\title{
Post-industrial systems of societies
}

In this special issue we introduce some selected contributions presented at the seminar The post-industrial societies dealing with complexity: knowledge to manage the knowledge society organised by the European Union for Systemics (EUS) and held on September 20, 2013, in Charleroi, Belgium. Charleroi was a former industrial area for the production of iron, steel and glass, located in the centre of a coal basin. The decline of the coal industry in the 70 has left Charleroi in a bad economic shape: its unemployment and poverty rates were one of the highest in Europe during the 80 and 90 . Since the early 2000, this ravaged area has opened itself to new horizons (health care, transportation and telecommunications) and reinvented itself. Therefore it represented a highly symbolic and inspiring metaphor for the topic of the seminar.

The seminar in general, and the contributions published here ,considered the shift from the industrial society to the post-industrial societies based on knowledge as primary resource.

However from the presentations and the discussion a new emergent understanding of the processes of such shift occurred, at different levels and in different context.

We try to summarise saying that since the focus was on the knowledge to be created and used to manage the complexity of post-industrial, knowledge societies, the interest was rather on outlining such complexity.

In doing so an interesting summarising emergent aspect emerged, that is, the double nature of post-industrial societies.

One internal, when sources of complexity are, for instance:

- augmented reality through simulations and multidimensional, simultaneous, and coherent information;

- data availability (so-called Big Data);

- delocalisation and globalisation;

- duplicability;

- ergodic-like change or trade-off between time and space;

- epiphenomena, i.e., secondary phenomenon occurring alongside or in parallel to the primary phenomenon;

- high general networked interconnections;

- high manipulability inventing and processing cognitive models;

- high virtuality;

- hyper connection; 
- importance of categories of individuality;

- instabilities of coherence rather than of equilibrium;

- inter-changeability;

- knowledge-intensiveness;

- simultaneous combinations of structured organisations and emergent phenomena;

- multiplicity;

- networked availability of knowledge;

- multiple non-linearity;

- on-line actions;

- products and services come with induction to use more than directions to use;

- interfaces;

- quick transformation of solutions into new problems;

- reduced time between design, implementation, and marketing;

- short general life span.

One external, since post-industrial societies are, for their nature, dynamical systems of different kind of societies not only interacting, but often simultaneous and superimposed combining, for instance, classical usages of new resources and technologies and non-classical usages of old resources such as in communications, transportations and knowledge management in services.

Like in complexity theory it is difficult to conceptually separate and consider phenomena and processes as independent, external like separated environments.

The main cognitive resource to deal with such phenomena and properties is systems science.

The classical pre-complexity systemic approaches focusing on properties like anticipation, automate, completeness, contextindependence, control, decision, forecast, growth, non-connectedness objectives, optimisation, organise, planning, precision, regulate, reversibility, separate, solve, and standardise, are not suitable because of limited effectiveness since any combination of them has the same systemic power lower than the one necessary to deal with of complexity.

Different systemic post-Bertalanffy understandings of secondgeneration have been considered in the articles published here like: Coworking spaces, Managing Principles, Multiple Systems, Networks Properties, and Plural agencies, all waiting for a possible future eventual 
general theoretical approach. They deal with complexity of social systems while examples of generic second generation systemic concepts focus on coherence, development rather than growth, dynamic usage of models to maintain coherences, emergence, entanglement, incompleteness, irreversibility, meta-structures, multiple non-homogeneous, multiplicity, non-linearity, non-symbolic, quasi, scenarios, self-organisation, simultaneity, uniqueness, uncertainty and incompleteness as resources.

The contributions presented here introduce, consider and try to apply this second-generation systemics to a specific kind of systems, i.e. socioeconomic systems of systems and their properties.

The contributions presented here not only exemplify the urgency of new suitable interdisciplinary post-Bertalanfy approaches but also are excellent examples of second order non-reductionism still allowed by the classical pre-complexity systemics when used to ineffectively deal with huge varieties of properties of complex systems non-reducible to generic noncomplex systems as it is the case for socio-economic systems.

A very new understanding of management and learning is required as challenge. 
